\title{
Auf dem Weg in die Scoringgesellschaft? Über den Umgang mit digitalen Statusdaten
}

\section{Zusammenfassung}

Der Beitrag beschäftigt sich mit der wachsenden Bedeutung von Scoringverfahren zur Bewertung und Klassifikation von Personen. ${ }^{2}$ Es wird argumentiert, dass durch die Digitalisierung nicht nur neue technologische Innovationen in die Gesellschaft Einzug halten, sondern dass es auch zur Datafizierung des Sozialen kommt, im Zuge derer immer mehr Lebensäußerungen und Aktivitäten in einheitliche Bewertungsraster überführt werden. Algorithmische Scoringverfahren werden genutzt, um Verhalten vorherzusagen, Risiken zu prognostizieren oder Handlungen zu steuern. Dabei kommt es einerseits dazu, dass Daten an Relevanz gewinnen, die außerhalb eines spezifischen Sachbereichs liegen, andererseits entstehen durch die Scores Kopplungen zwischen unterschiedlichen Teilsystemen. Der Beitrag problematisiert diese Entwicklungen und fragt nach potenziellen soziologischen Folgen.

In diesem Beitrag soll eine dezidiert soziologische Perspektive auf Scores und ihre Erstellung eingenommen werden. Es stehen also weniger einzelne Technologien oder Algorithmen im Vordergrund, sondern die Frage, welche gesellschaftlichen Effekte diese Bewertungsinstrumente erzeugen.

Das gegenwärtig wohl berühmteste und auch in diesem Band breit rezipierte Beispiel ist das chinesische Social-Credit-System. Bis zum Jahre 2020 soll dort jeder Bürger mit einem Vertrauensscore ausgestattet werden, der darüber Auskunft gibt, wie er sich als Mitglied der Gesellschaft darstellt. In den Score fließen Informationen über Verkehrsdelikte, Konsumverhalten oder überzogene Ausleihfristen in der Bibliothek ebenso ein wie das Verhalten im Internet, Aktivitäten in den sozialen Medien oder Bewertungen von Arbeitgebern und Vermietern. Mit Hilfe großer Internetfirmen sollen all diese Daten sukzessive zu einem integrierten Datensatz zusammengefasst werden. In mehr als 50 Städten und Gemeinden werden Vorläufer dieses Systems bereits als Pilotprojekte getestet - so beispielsweise in Shanghai, wo eine App, die bisher noch freiwillig ist, Informationen von 97 Ämtern abfragt und insgesamt fast 5.200 Einzeldaten

2 Der Text ist unter Verwendung der männlichen Form für Berufs- und Personengruppen und -bezeichnungen verfasst. Geneint sind Personen aller Geschlechteridentität gleichermaßen. 
bündelt. Personen mit hohem Score erhalten Vorteile und können z.B. in der Bibliothek ohne Kaution Bücher ausleihen. Gleichzeitig gehen mit niedrigem Score Sanktionen wie „Travel Bans“, also Verbote, bestimmte Transportmittel oder interregionale Züge zu nutzen, Visarestriktionen oder erschwerter Zugang zu Hotels, Reisen, Wohnungen oder Studienkrediten einher. Die chinesische Regierung gibt an, mit dem Score „soziales Vertrauen“ schaffen zu wollen. Angesichts wachsender Korruption und tiefer gesellschaftlicher Verwerfungen scheint dieses Mittel jedoch eher dazu zu dienen, durch Überwachung soziale Kontrolle auszuüben.

Die Studie von Genia Kostka (2019) ist die erste mir bekannte repräsentative Studie überhaupt, die sich mit der Akzeptanz solcher sozialer Scorings in China beschäftigt. Darin wird deutlich, dass eine überwältigende Mehrheit von über 80 Prozent der Befragten Scoring-Systeme gut oder sehr gut finden, 19 Prozent stehen diesen indifferent gegenüber und nur 1 Prozent der Befragten lehnt Scores grundsätzlich ab. Besonders bemerkenswert ist zudem die sozialstrukturelle Verteilung der Zustimmung: Die höheren Schichten, also die Einkommensstarken und gut Ausgebildeten sehen Scoringverfahren nicht etwa skeptisch, sondern haben eine etwas stärker affirmative Haltung zu ihnen als die unteren Schichten. Scheinbar sind Konzepte von Privatheit oder des Schutzes von privaten Informationen in der chinesischen Kultur nicht weit verbreitet.

China ist sicherlich ein extremes Beispiel, allerdings befinden sich auch in westlichen Gesellschaften digitale Bewertungssysteme in ganz verschiedenen Feldern auf dem Vormarsch. In den USA werden Scorings bereits in der Sozialhilfe eingesetzt und die Niederlande planen die Einführung von Punktesystemen in der Arbeitsmarktpolitik, um Individuen zu klassifizieren und ihnen dann bestimmte Möglichkeiten einzuräumen und andere zu verwehren. In kommunalen Verwaltungen und dem öffentlichen Dienstleistungsbereich werden Daten integriert, um Ansprüche festzusetzen, „Risikofamilien“ zu identifizieren oder Leistungsmissbrauch festzustellen. Bei der Polizei probt man die Verwendung von „Risikoscores“, um die Polizeiarbeit effektiver zu machen. Wer in den USA einen Babysitter über das Internet sucht, wird immer wieder mit dem Hinweis konfrontiert, dass es sehr gefährlich sei, eine völlig unbekannte Person ins Haus zu holen und ihr auch noch die eigenen Kinder anzuvertrauen. Über Scoring- und Ratingverfahren kann nunmehr die Vertrauenswürdigkeit ermittelt werden. Bis vor kurzem konnte man sich auf der Webseite MyLife.com mithilfe des Namens und des Wohnortes der Person einen Reputationsscore für alle erwachsenen und gemeldeten Amerikaner ausgeben lassen. Dieser Reputationsscore geht in mehreren Schritten vor: Zunächst werden alle Namen und Aliasnamen einer Person, also auch etwaige Namensänderungen bei Heirat, festgestellt. Dann ruft die Seite alle Adressen und Telefonnummern sowie sämtliche Einträge in sozialen Medien, inklusive derer, die gelöscht wurden und sich 
technisch wiederherstellen lassen, ab. Zudem werden Daten zur erweiterten Familie und zum Freundeskreis verarbeitet. Damit jedoch nicht genug: Auch registrierte Lizenzen, Waffenbesitzgenehmigungen inklusive Lizenznummer und Ablaufdatum, frühere und anhängige Gerichtsverfahren, Geschwindigkeitsübertretungen, Beschreibung der zur Last gelegten Straftaten, Privatinsolvenzen oder Sittlichkeitsdelikte werden einbezogen, um schließlich einen Score zwischen 0 (negativ) und 5 (positiv) auszugeben, dazu eine Anzeige auf einem Vertrauenstacho von Rot bis Grün. Die Nutzer sind begeistert, so findet man auf der Website Kommentare von Luisa oder Ricardo:

„You'd be crazy to rent someone an apartment not looking at the background report first. It saved me tons of trouble."

„I declined a job offer after I did a background report check on the guy who was going to be my supervisor.“

Und noch wichtiger ist natürlich Larry:

„I felt a lot better about my daughters new boyfriend after I looked at the reputation score."

Die zentrale Frage ist: Worum handelt es sich eigentlich bei diesen Scorings? Scoringtechniken oder Scoringverfahren sind neuartige Techniken der Klassifizierung, die auf Differenzmessung und spezifischen Arten von Bewertungen aufbauen. Der englische Begriff „Score“ bedeutet zunächst Punktestand und „Scoring“ heißt dann Punktwertermittlung. Das sind Technologien oder Techniken, die in unterschiedlichen Feldern schon immer eine Rolle gespielt haben. Wer Golf spielt, weiß, dass es eine Scorecard gibt. In Unternehmen dient eine Balancescorecard als Erfassungsbogen aller Aktivitäten und Leistungsströme.

An dieser Stelle soll der Fokus allerdings auf der Bewertung von Individuen liegen. Scoren kann man vieles: Gesundheit, Vertrauen, Risiko, Aktivitäten, Popularität, politische Loyalität, Mobilitätsverhalten, Rentabilität, Bonität etc. Beim Scoring handelt es sich um einen Vorgang der Erzeugung und Visibilisierung von Differenz. Das bedeutet, dass durch einen numerischen Punktestand Dinge als besser oder schlechter, mehr oder weniger, oben oder unten verstanden werden können. Sie werden in eine Rangfolge, in eine Hierarchie gebracht. $\mathrm{Da}$ viele dieser Scorings öffentlich sind, geht es letztendlich auch um die $\mathrm{Zu}-$ weisung von gesellschaftlicher Reputation.

Soziale Reputation lässt sich als Form des Sozialprestiges oder als soziales Kapital verstehen, das einer Sache oder einer Person einen bestimmten Wert zuschreibt, der sich jeweils auf einen evaluativen Standard bezieht. Die Vielfalt der zur Verfügung stehenden Informationen führt mitunter dazu, dass nicht mehr vollständig nachvollziehbar ist, auf welche Bewertungsbasis eigentlich Bezug genommen wird, wie im genannten Fall. Ob es beispielsweise ein Verkehrsdelikt ist oder eine irgendwo nicht gezahlte Gebühr, die dazu führt, dass 
die Babysitterin besser oder schlechter abschneidet, ist nicht immer erkennbar. Durch viele der Scoringverfahren wird ein symbolischer Raum ungleicher Positionen in der Reputationsverteilung aufgespannt. Die Positionierung in diesem Raum sorgt dann für handfeste Konsequenzen. Jemand, der bei diesen Bewertungssystemen nur eine zwei oder eine eins hat, wo alle anderen eine vier oder eine fünf haben, der wird eben nicht so ohne weiteres als Babysitter engagiert werden. Die beiden amerikanischen Forscher Fourcade und Healy (2017) sprechen in Anlehnung an Bourdieu von Überkapital. Sie beschreiben damit eine auf Daten beruhende Kapitalform, in welcher Informationen über Individuen gespeichert und dann dazu genutzt werden können, um Personen zu klassifizieren und zu bewerten und damit schlussendlich über die Zuteilung von Marktoder Lebenschancen zu entscheiden.

Im Folgenden versuche ich, die soziale Funktionsweise von Scorings an einigen Beispielen zu verdeutlichen. Zunächst soll es um Kredit-Scorings gehen, die schon relativ weit verbreitet und auch bekannt sind. Natürlich wurden Kunden von Banken immer im Hinblick auf ihre Bonität oder ihre Kreditwürdigkeit bewertet. Auch Massendaten spielten dabei eine wichtige Rolle, verbreitern diese doch die Informationsgrundlage, schaffen mehr Vergleichsmöglichkeiten und ermöglichen so genauere Vorhersagen über potenzielle Kreditausfälle. Mit der Digitalisierung wird nun immer mehr erfassbar und für die Risikokalkulation nutzbar gemacht. Dabei kommt es auch dazu, dass Daten aus weit von den Finanzangelegenheiten entfernten Bereichen für die Zuweisung eines KreditScores relevant werden können.

Ein sehr eindrucksvolles Beispiel dafür, wie sich die Informationsbasis dieser Kredit-Scorings verändert hat, ist die Moven Bank in New York, die nur als Online-Bank funktioniert und einen aktuellen Echtzeit-Kredit-Score für all ihre Kunden anbietet. Dieser Score bezieht eben nicht mehr nur die klassischen Informationen Wohnort, Berufsstand, Einkommenshöhe oder mögliche Sicherheiten ein, sondern stützt sich stark auf öffentliche Informationen, Registerdaten, amtliche Daten und Daten aus den sozialen Medien. Die Einbeziehung sozialer Medien bedeutet, dass der eigene Kredit-Score sehr stark davon abhängt, welchen Freundeskreis, welches Netzwerk eine Person hat. Wenn demnach jemand im sozialen Umfeld einer Person insolvent wird, führt das dazu, dass sich auch der Kredit-Score reduziert und sich die individuellen Konsumchancen verringern. Tatsächlich gibt es gute Gründe für ein solches Scoring-System, denn die prädiktive Qualität dieses Scores in Bezug auf tatsächliche Kreditausfälle ist hoch. Die prognostische Qualität dieser Daten ist besser, als wenn man nur die Straße, den Wohnort, das Einkommen und möglicherweise den Arbeitgeber für die Berechnung einer Ausfallwahrscheinlichkeit zugrunde legt. Die relative Güte dieses Scores wird als Begründung dafür genommen, dass man auf immer 
weitere Informationen über Personen, ihre Netzwerke und ihr Verhalten zurückgreift.

Einerseits werden nun also ganz unterschiedliche Lebensäußerungen für die Berechnung dieses Scores datenmäßig ausgewertet, andererseits finden die Scores auch in immer weiteren Bereichen Verwendung, für die sie ursprünglich nicht gedacht waren, etwa bei Versicherungsabschlüssen, auf dem Wohnungsmarkt, beim Mietwagenverleih oder bei der Personalrekrutierung. Auch in diesem Feld sind die USA wahrscheinlich ein Extrembeispiel, aber auch in anderen Ländern lässt sich dies beobachten. Die ursprünglich auf den Finanzsektor beschränkten Kredit-Scores schwappen also nach und nach auf viele andere Felder über. Das geht bis hin zu Dating-Websites, auf denen Menschen ihren Kredit-Score ausweisen. Schließlich ist es für den potenziellen Partner auch interessant zu wissen, ob das Gegenüber in der Lage sein wird, einen Kredit aufzunehmen oder sich ein Eigenheim zu leisten. Viele Informationen über Individuen sind nicht ohne weiteres zu bekommen: Ein Kredit-Score kann dabei eine relevante Information werden, weil von finanzieller Sicherheit oder Gleichheit in der Partnerschaft auch deren Stabilität abhängt. In China gibt es viele Menschen, die sich selbst auf ihren Facebook-Seiten mit diesen Informationen bewerben. Hier erkennt man ein Umnutzen einer einstmals ausschließlich in der Interaktion zwischen Finanzdienstleister und Kunde relevanten Größe.

Kommen wir zum Gesundheitsscoring. Das Verhältnis zwischen Arzt und Patient ist stets ein sehr intimes, ein Vertrauensverhältnis. Angefangen bei den Entwicklungen von elektronischen Gesundheitsakten über die Nutzung digitaler Tools der Selbstvermessung bis hin zur Etablierung von Gesundheitsscores sowohl bei den Krankenkassen, im betrieblichen Gesundheitsmanagement als auch in Eigenregie, spielen Daten eine immer größere Rolle. Von verschiedenen Anbietern werden nunmehr Gesundheitsscores zur Verfügung gestellt. Was ändert sich dadurch im Gesundheitsverhalten oder in der Wahrnehmung von Gesundheit? Bei einem klassischen Arztbesuch gibt es nur die binäre Kodierung zwischen „gesund“ und „krank“, die sich in einer „Gesund-“ oder „Krankschreibung" ausdrückt. Gesundheitsscores hingegen nutzen metrische Skalen mit einer großen Zahl von Abstufungen. Gesundheit wird somit zu einem Gut, von dem man nicht genug bekommen kann. Wenn der Score zwischen 0 und 1.000 liegt, dann mag ein Individuum bei 600 vollkommen gesund sein, aber es wäre natürlich attraktiver auf 650 oder $680 \mathrm{zu}$ kommen. Wenn jemand aber mal bei 680 war, dann mögen 580 zwar im sozialen Vergleich noch nicht schlecht sein, aber es würde einen individuellen Rückschritt bedeuten und somit auch eine Verhaltensaufforderung zu mehr gesundheitsbezogener Aktivität enthalten. Derzeit unterscheidet sich die Art und Weise, wie Gesundheitsscores erstellt werden, noch stark. Der Score des Schweizer Unternehmens „Dacadoo“ liegt zwischen 1 und 1.000 und bezieht neben Körper- und Vitaldaten auch das 
Wohlbefinden und den Lebensstil ein - greift also auch hier wieder auf Informationen zurück, die die Lebensweise allgemein betreffen. In der Selbstbeschreibung des Unternehmens findet sich zum Score folgende interessante Analogie. Der Score sei

„wie ihr eigener persönlicher Aktienkurs Ihrer Gesundheit und Ihres Wohlbefindens in Echtzeit, ähnlich wie bei einem Aktienkurs an der Börse.“ (zit. nach Mau 2017: 116)

Auch hier geht es wieder um Echtzeitbeobachtungen: Alle Veränderungen können registriert werden und sollen dann reflexiv auch auf das Verhalten von Individuen zurückwirken. Auch wenn diese Gesundheitsscores nicht im engeren Sinne öffentlich sind, so können sie durchaus durch Dritte eingesehen werden, beispielsweise durch betriebliche Krankenkassen, mit denen Dacadoo zusammenarbeitet. Es gibt immer mehr Unternehmen, die im Rahmen ihres betrieblichen Gesundheitsmanagements auf Daten von Beschäftigten zurückgreifen. Aus der Sicht des Arbeitgebers erweitern sich die Möglichkeiten der Steuerung von Individuen dadurch enorm, denn nun können sie aktiviert und an gesünderes Verhalten herangeführt werden.

Auch für die Krankenversicherer sind die Daten der Gesundheitsscores höchst interessant, weil sie die Möglichkeit der Etablierung individualisierter Tarifsysteme bieten. Wenn bei jedem Schritt Punkte gesammelt werden können, bei jedem Mal um den Block laufen oder den Hund ausführen, dann sind dies dauerhafte Beobachtungssysteme, die sehr stark das individuelle Verhalten regulieren und Rückwirkungen erzeugen können. Die Versicherungen träumen davon, dass dies zukünftig in Boni- und Statussysteme mündet, wie man sie z.B. aus dem Flugverkehr kennt. Es gäbe dann Bronze-, Silber-, Gold-, Platinoder sogar Diamant-Kunden bei Versicherungen, die sogar in unserem Alltag eine Rolle spielen sollen, wie es sich ein Generali-Manager vorstellt:

„Wenn da jemand auf der Party sagt, dass er den Diamantstatus hat, gilt er als cooler Typ“ (zit. nach Wanner/Fromme 2016: 19).

Bei dieser Vorstellung spielt der Reputationswert eines Scores wiederum in die sozialen Verhältnisse mit hinein. Es handelt sich entsprechend um Systeme der Ermittlung sozialer Wertigkeit, die als symbolisches Kapital oder als Generatoren symbolischen Kapitals wirksam werden können. Dieses Kapital ist konvertierbar, d.h. es kann auch in anderen Feldern benutzt werden. Es ist heute schon erkennbar, dass die in den verschiedenen Bereichen des Lebens gesammelten Punkte und Status-Scores, sei es die finanzielle Bonität, Sympathiebekundungen durch Freunde oder Gesundheitsscores, auch in andere Bereiche hineinschwappen. Die Soziologie ist eng mit dem Theorem der funktionalen Differenzierung verbunden, das letztlich besagt, dass wir in unterschiedlichen Feldern 
jeweils eigene Operationslogiken vorfinden. Die Scores schaffen aber neue Übertragungsmöglichkeiten. Das chinesische Beispiel zeigt etwa, wie auf Grundlage von Daten sogar ein vereinheitlichtes Bewertungssystem entsteht. Soweit sind westliche Staaten nicht, aber auch hierzulande diffundieren die internen Logiken und Rationalitäten einzelner Felder in andere Bereiche, sodass subsystemspezifische Leitunterscheidungen zum Teil unterlaufen oder überlaufen werden. Durch die Verwendung von Zahlen, Numeriken und Punkten kommt es zu einer Verstärkung des Grenzverkehrs zwischen unterschiedlichen Subsystemen. Verständlicherweise kann für einen Arbeitgeber der Gesundheitsscore eines Individuums höchst relevant sein. Dadurch kann bei der Einstellung neben dem Humankapital auch das Gesundheitskapital ermittelt werden, das Aufschluss darüber gibt, wie lange das Humankapital genutzt werden kann und mit welchen Risiken die Beschäftigung verbunden ist. Gleichermaßen kann es für einen Vermieter eine Rolle spielen, welchen Reputationsscore eine Person hat, weil daraus geschlussfolgert wird, ob es sich um eine verlässliche Person handelt.

Viele Menschen glauben, dass mit diesen Statusdaten und Score-Systemen eine gerechtere Gesellschaft auf uns zukommt, weil sich dadurch Statuszuweisungsprozesse rationalisieren: Subjekte würden als die erkannt und behandelt, die sie wirklich sind, und nicht zu großen Risikogruppen zwangsklassifiziert und mit Leuten zusammengeworfen, mit denen sie gar nichts zu tun haben. Diese Sichtweise verkennt allerdings, wie weit unsere (Sozial-)Versicherungen davon abhängen, dass wir absichtlich und willentlich von individuellen Differenzen abstrahieren. Dies gilt beispielsweise für die Rentenversicherung: Die müsste eigentlich, wenn man nach Gerechtigkeitsgesichtspunkten geht, nach sozialen Klassen und nach Berufen differenzieren, weil es z.B. keine Steigerung der Lebenserwartungen bei den unteren 20 Prozent der Einkommensverteilung gibt. Das heißt, die Lebenserwartung verlängert sich vor allem für höhere Einkommensgruppen, die dann auch deutlich länger Leistungen aus der Rentenversicherung beziehen können. Gleiches gilt für Unterschiede zwischen den Geschlechtern. Auch hier hat sich die Gesellschaft darauf geeinigt, keine Differenzierungen zu machen, weil diese sozial als Diskriminierung verstanden werden können. Mit dem Wachstum der Daten kommt es zu immer weiteren Differenzierungsmöglichkeiten. In diesem Sinne meinen Datafizierung und Digitalisierung auch Spaltbarkeit des Sozialen, also eine Form der Hyperindividualisierung, die uns alle unterscheidbar und die Gesellschaft immer granularer und erkennbarer macht, weil eben alle Aspekte des Lebens in dieses Datenregime einbezogen werden.

Was man beobachten kann, ist ein Überschwappen dieser Arten von Bewertungspraktiken in den sozialen bzw. gesellschaftlichen Bereich hauptsächlich bei der Bewertung von Individuen, aber auch von Dienstleistungen, Pro- 
dukten oder Unternehmen. Erste Studien in den USA wie die von Virginia Eubanks (2018) zeigen, wie in einigen Bundesstaaten z.B. im Bereich der Sozialhilfe solche neuen Scoring-Systeme eingesetzt werden, die dann dazu führen, dass Personen in ganz unterschiedlichen Feldern exkludiert werden. Der Score weist weit über den Bezug von Sozialhilfe hinaus: Menschen sind nicht mehr in der Lage, eine Wohnung oder einen neuen Arbeitgeber zu finden, weil diese Informationen weitergegeben werden. Die Autorin kommt zu dem Schluss, dass sich digitale Armenhäuser entwickeln, also Personen systematisch aus unterschiedlichen Gesellschaftsbereichen ausgeschlossen werden.

Diese eher skeptischen und etwas düsteren Beobachtungen sind zunächst einmal nur eine Problematisierung, die auch aus einer regulatorischen Perspektive beleuchtet werden kann (vgl. auch den Beitrag von Roßnagel in diesem Band). Deutlich sollte werden, dass sich mit dem Voranschreiten der Scorings neue Formen der statusbezogenen Klassifizierung herausbilden. Die All-Verfügbarkeit der Daten für immer mehr Bereiche des sozialen Lebens bringt es letzten Endes mit sich, dass die Zuweisung von und die Kommunikation über Status immer stärker auf messbaren, quantifizierbaren Aspekten beruht. Noch ist nicht ausgemacht, welche Zügel sich dieser neuen Scoring-Gesellschaft anlegen lassen, aber es gibt gute Gründe anzunehmen, dass sich das Regime der Zahlen noch längst nicht zu Ende entwickelt hat, und dass mit dem Voranschreiten der Digitalisierung auch immer neue Möglichkeiten entstehen werden, Scores zur Ermittlung von Wertigkeit einzusetzen.

\section{Literatur}

Eubanks, V. (2017): Automating Inequality. How high-tech tools profile, policy, and punish the poor. New York: St. Martin Press.

Marion, F. / Healy, K. (2017): Seeing Like a Market. Socio-Economic Review 15: 9-29.

Kostka, G. (2019, im Erscheinen): China's Social Credit Systems and Public Opinion: Explaining High Levels of Approval, New Media \& Society.

Mau, S. (2017): Das metrische Wir. Über die Quantifizierung des Sozialen: Berlin: Suhrkamp.

Wanner, C. / Fromme, H. (2016): Läuft bei Generali. In: Süddeutsche Zeitung, 21. Juni, S. 19. 